TITRE: ASSURER LA RIGUEUR SCIENTIFIQUE DE LA RECHERCHE-ACTION

Auteur(s): LOUISE BOURGEOIS, UNIVERSITÉ LAURENTIENNE

PublicATION: LA RECHERCHE-ACTION ET LA RECHERCHE-DÉVELOPPEMENT AU SERVICE DE LA LITTÉRATIE.

PAGES: $6-20$

DiRecteurs: ISABELle CARIgnAN, MARIE-Christine BeAudry ET FrançOIS LAROSE ÉdiTEUR: LES ÉdITIONS DE L'UNIVERSITÉ DE SHERBROOKE, 2016.

ISBN: 978-2-7622-0354-7

URI: HTTP://HDL.HANDLE.NET/11143/8807

DOI: HTTP://DX.DOI.ORG/10.17118/11143/8807 


\section{Assurer la rigueur scientifique de la recherche-action}

Louise Bourgeois, EAO, M.Éd., doctorante à l'Université d'Ottawa

Professeure à l'Université Laurentienne

Résumé : Les critères auxquels on peut se référer pour juger de la qualité et de la rigueur d'une recherche varient selon la posture épistémologique des chercheurs. Alors que la recherche positiviste s’appuie sur des critères de validité scientifique et que la recherche interprétative est soutenue par des critères méthodologiques et relationnels, qu'en est-il de modèles de recherche comme la recherche-action? Cet article vise d'abord à rappeler les critères de rigueur de la recherche interprétative, à faire ressortir les critères qui s'appliquent spécifiquement à la recherche-action et, enfin, à proposer des moyens pour satisfaire à chacun de ces critères.

Mots-clés : critères de scientificité, rigueur, recherche-action, moyens. 
La question de la valeur scientifique d'une recherche préoccupe tous les chercheurs, et ce, peu importe leur posture épistémologique. Alors que les critères de scientificité de la recherche positiviste sont bien ancrés dans la validité scientifique, ceux de la recherche interprétative relèvent davantage de la rigueur méthodologique et relationnelle (Anadón, 2006; Gohier, 2004; Lincoln, 1995). Mais qu'en est-il de modèles de recherche comme la recherche-action qui, bien qu'elle vise la production de savoir, entretient également des visées de changement dans la pratique professionnelle? Cet article vise deux objectifs : 1) il cherche à rappeler les critères de rigueur méthodologique et les critères relationnels qui s'appliquent à toutes les recherches qui se situent dans le giron qualitatif et 2) il a également pour but de faire ressortir les critères qui visent spécifiquement à soutenir la crédibilité des résultats de la recherche-action. Par ailleurs, pour chacun de ces objectifs, il est également question d'évoquer des exemples de mécanismes à mettre en place dans la recherche pour satisfaire a) les critères de rigueur méthodologiques (crédibilité, transférabilité, fiabilité et constance interne), b) les critères de rigueur relationnels (équilibre et authenticité) et c) les critères propres à la rechercheaction en fonction des modèles adoptés (respect des valeurs et des principes démocratiques, faisabilité, appropriation et cohérence systémique).

\section{Critères de rigueur méthodologique}

C'est dans le contexte du renouveau du paradigme interprétatif des années 1950 qu'ont commencé à émerger des critères de scientificité propres aux méthodes qualitatives (Laperrière, 1997). Le milieu du $20^{e}$ siècle a marqué une période pendant laquelle les tenants du paradigme interprétatif cherchaient à redéfinir les critères de validité du positivisme conventionnel dans le but de tenir compte des caractéristiques particulières de la recherche interprétative. En effet, le but positiviste de chercher à neutraliser la subjectivité dans la recherche n'était pas pertinent à la recherche interprétative justement parce que celle-ci adhère à une position selon laquelle la connaissance est subjective puisqu'elle est liée au vécu du sujet (Guba et Lincoln, 2005). Ainsi, Lincoln et Guba (Guba, 1981; Lincoln et Guba, 1985) ont élaboré des critères méthodologiques parallèles aux critères de validité du positivisme qui visent avant tout à tenir compte de la subjectivité de la recherche interprétative et des effets de l'interaction entre le chercheur et le sujet (Laperrière, 1997). Laperrière (1997) explique que:

[...] les chercheurs qualitatifs voulaient maximiser la validité de leurs résultats en balisant ou en exploitant les ressources de la subjectivité plutôt qu'en essayant de l'exclure des processus de recherche, puis en encourageant une étude des phénomènes sociaux en contexte naturel plutôt que dans un contexte qu'on tentait de neutraliser au départ pour en arriver à établir rapidement des généralisations. (p. 367)

Quatre critères liés aux méthodes et aux procédures d'analyse de la recherche interprétative ont été définis en parallèle à ceux du paradigme positiviste. Il s'agit des critères de crédibilité, de transférabilité, de fiabilité et de constance interne (Lincoln et Guba, 1985). 


\section{Crédibilité}

Parallèlement à la validité interne qui vise à vérifier la capacité de la recherche positiviste à produire des données attribuables à l'intervention plutôt qu'à d'autres phénomènes (Cohen, Manion et Morrison, 2011), le critère de crédibilité en recherche interprétative s'intéresse à vérifier la congruence entre le sens véhiculé par le sujet et le sens dégagé par le chercheur, notamment dans la collecte, l'analyse et l'interprétation des données (Gohier, 2004; Lincoln et Guba 1985; Savoie-Zajc, 2011). Au bout du compte, le critère de crédibilité vise à répondre à la question : sommes-nous devant un portrait authentique de ce qui a été observé? Alors que l'engagement prolongé du chercheur sur le terrain, la triangulation des méthodes et le journal réflexif du chercheur sont toutes des techniques qui permettent de soutenir la crédibilité des résultats de la recherche, la vérification du texte de la recherche par les sujets est considérée par Lincoln et Guba (1985) comme la plus importante stratégie pour établir la crédibilité.

\section{Transférabilité}

Une importante visée du paradigme positiviste est de développer et de vérifier des règles généralisables à un grand nombre de contextes différents (Cohen, Manion et Morrison, 2011). Il s'agit de la validité externe. En recherche interprétative toutefois, l'échantillon d'étude n'est généralement pas statistiquement représentatif et, par conséquent, les résultats ne mènent pas à des généralisations (Guba, 1981). Ainsi, le critère de transférabilité vise à déterminer si les conclusions d'une recherche peuvent avoir un sens dans un autre contexte que celui étudié (Gohier, 2004; Pourtois et Desmet, 2007). Ce critère se partage entre le chercheur et celui qui cherche à utiliser les résultats de la recherche dans son milieu. II revient alors au chercheur de fournir des descriptions riches du contexte et de l'échantillon de l'étude et à l'utilisateur de s'interroger sur la pertinence et la ressemblance entre le contexte de la recherche et son propre milieu (Savoie-Zajc, 2011).

\section{Fiabilité}

L'objectivité en recherche quantitative, qui est liée à la neutralité des données observables et mesurables, revient en recherche interprétative à la fiabilité qui vise à démontrer la transparence du chercheur et, par le fait même, l'indépendance de ses interprétations par rapport à ses biais (Gohier, 2004; Pourtois et Desmet, 2007). À la base de ce critère « se trouve la lucidité du chercheur à l'égard de ses jugements et la reconnaissance de ceux-ci en tant qu'éléments influençant ses analyses et interprétations (triangulation interne du chercheur) (Mucchielli, 2009, p. 60).

Pour assurer la fiabilité de la recherche, la triangulation s'avère encore une fois la stratégie la plus utile, notamment la triangulation des observateurs ainsi que la triangulation interne du chercheur (Pourtois et Desmet, 2007). Le chercheur doit démontrer qu'il est conscient de sa subjectivité (Miles et Huberman, 2003) et prendre des mesures pour attester que les résultats émergent des données 
et non de ses intérêts ou de ses motivations. La collaboration de plusieurs chercheurs dans la recherche qui peuvent comparer leurs observations et leurs interprétations des données peut soutenir la fiabilité de la recherche. La vérification interjuges, par exemple, peut être utilisée pour assurer la fiabilité des résultats où les divergences entre les codeurs sont analysées et résolues par l'intermédiaire d'une analyse conjointe du matériel. À cet effet, Miles et Huberman (2003) recommandent un indice de fidélité d'au moins 70 \% avant les ajustements et de 90 \% après. Bien qu'il n'y ait pas d'indicateurs dans la littérature quant au nombre de codeurs à utiliser dans le processus, Kolbe et Burnette (1991) ont remarqué, dans une recension de recherches, qu'il s'agit le plus souvent de deux codeurs. De la même façon, il n'y a pas d'indicateurs dans la littérature quant au pourcentage du matériel qui devrait être recodé par différents codeurs pour assurer la fiabilité. Quand cette donnée est fournie dans les recherches interprétatives, on peut observer qu'elle peut varier entre $10 \%$ jusqu'à la totalité du corpus du matériel qui est recodé par au moins deux codeurs. Dans la littérature sur la fiabilité du codage, la formation et l'indépendance des juges semblent être une plus grande préoccupation.

\section{Constance interne}

Le critère de fidélité en recherche quantitative vise la stabilité et la reproductibilité des résultats (Cohen, Manion et Morrison, 2011); il a peu de sens dans un cadre qualitatif où le changement est fondamental. Si la fidélité, en recherche quantitative, vise la persistance d'une procédure de mesure à procurer la même réponse, la constance interne, en recherche interprétative, revient plutôt à "l'indépendance des observations et des interprétations par rapport à des variations accidentelles ou systématiques » (Gohier, 2004, p.7). En effet, la constance interne cherche à fournir un degré d'assurance que les résultats de la recherche n'ont pas été influencés, par exemple, par la personnalité du chercheur, les instruments utilisés ou les conditions de collecte de données (Mucchielli, 2009). Pour augmenter la constance interne de la recherche, Pourtois et Desmet (2007) citent de nouveau la triangulation, plus précisément la triangulation des chercheurs, où au moins deux chercheurs collaborent à toutes les étapes de la recherche, ainsi que la triangulation temporelle, où le chercheur tient compte de l'évolution du phénomène dans le temps. À défaut d'autres chercheurs participant à la recherche, la vérification par un chercheur externe peut servir aux fins de la triangulation des chercheurs (Creswell, 2007; Miles et Huberman, 2003).

Enfin, ces quatre critères (crédibilité, transférabilité, fiabilité et constance interne) visent à soutenir la rigueur méthodologique de la recherche interprétative, mais ils ne tiennent pas compte du caractère « dynamique, collaboratif et socioconstructiviste de l'approche interprétative » (Savoie-Zajc, 2000, p.171). Pour cette raison, des critères relationnels s'ajoutent aux critères méthodologiques dans le but de soutenir la qualité globale de la recherche puisqu'ils font davantage référence aux rapports qu'entretient le chercheur avec les sujets. 


\section{Critères relationnels}

Les critères relationnels élaborés par Guba et Lincoln (1989) n’ont pas d'équivalents en recherche quantitative puisqu'ils misent sur les relations qui s'établissent entre le chercheur et le sujet. Il s'agit du critère d'équilibre et des critères d'authenticité ontologique, éducative, catalytique et tactique. Manning (1997) souligne l'importance des critères relationnels pour soutenir la valeur de la recherche interprétative :

If the research fails to meet these commitments [balance of perspectives, learning by the researcher and respondents, shared knowledge and social action], the quality of the research (e.g. meaningfulness, usefulness, ability to enact social change) is questionable. (p. 94)

\section{Équilibre}

L'équilibre consiste à s'assurer que les points de vue de l'ensemble des sujets concernés par la recherche sont exprimés de façon équitable (Denzin et Lincoln, 2000; Gohier, 2004; Savoie-Zajc, 2011). Cet équilibre sous-entend que le chercheur doit fournir l'occasion, voire convaincre les sujets, d'exprimer leurs idées et en tenir compte, qu'il soit en accord ou en désaccord avec celles-ci. Savoie-Zajc (2011) mentionne que l'équilibre exige une certaine pondération des divers points de vue dans le texte. En effet, le critère d'équilibre cherche à amener le chercheur à se poser deux questions fondamentales:

1. Est-ce que tous les sujets ont eu l'occasion d'exprimer leurs idées pendant la recherche?

2. Est-ce que les points de vue de l'ensemble des sujets sont exprimés de façon équitable dans la recherche?

Pour qu'il y ait équilibre, Guba et Lincoln (1989) suggèrent des stratégies telles que la vérification du texte de la recherche par les sujets, l'implication de plus d'un chercheur à toutes les étapes de la recherche et l'engagement prolongé des chercheurs sur le terrain dans le but d'établir des rapports positifs avec les sujets.

\section{Authenticité}

L'authenticité se répartit en quatre critères : l'authenticité ontologique, l'authenticité éducative, l'authenticité catalytique et l'authenticité tactique.

L'authenticité ontologique vise à démontrer que les sujets ont amélioré leurs connaissances et élargi leurs représentations à propos de l'objet ou du phénomène à l'étude (Gohier, 2004; Savoie-Zajc, 2011). Cette transformation amène les participants à se construire des représentations plus riches et complexes de la réalité (Gohier, 2004). On peut penser, par exemple, à une entrevue où les sujets sont 
confrontés à des questions auxquelles ils n’ont jamais pensé et sont obligés de formuler de nouvelles idées pour y répondre.

L'authenticité éducative vise l'approfondissement de la connaissance de soi et une meilleure compréhension des représentations des autres participants (Gohier, 2004; Savoie-Zajc, 2011). La recherche oriente premièrement le sujet vers une prise de conscience de sa propre position pour ensuite mener à la comparer à celles des autres. L'intention est de faire en sorte que tous les participants acquièrent une compréhension plus riche, plus profonde et plus nuancée de leurs représentations et de celles des autres.

L'authenticité catalytique mise sur les apprentissages suscités par la recherche et vise des retombées au niveau du désir et du pouvoir d'action des sujets (Gohier, 2004; Savoie-Zajc, 2011). La recherche, par sa pertinence, devient ainsi un catalyseur qui incite à l'action. Ce critère encadre la recherche qui aborde un problème authentique, fait en sorte que sa finalité ne se limite pas qu'à la production de savoirs et met en place les conditions pour que l'engagement des sujets et du chercheur dépasse le contexte de la recherche.

L'authenticité tactique fournit les outils conceptuels aux participants qui leur permettent de passer à l'action (Gohier, 2004; Savoie-Zajc, 2011). Selon ce critère, le chercheur reconnait que les sujets qui participent à une recherche n'ont pas tous les mêmes habiletés et compétences. II s'engage ainsi à les former, selon leurs besoins et leurs désirs, à des formes spécifiques d'action politique ou sociale. Il s'agit d'une façon de tenter de rétablir un équilibre social dans le but de donner aux sujets les moyens et le soutien pour être en mesure de produire un changement.

Ces critères d'authenticité se vérifient généralement grâce aux témoignages des sujets au cours et au terme de la recherche. Le chercheur peut, par exemple, avoir recours aux témoignages des sujets attestant de l'élargissement de leurs connaissances, de l'amélioration de leurs pratiques ou de l'acquisition d'outils les habilitant à passer à l'action, mais il doit également être sensible et attentif à la qualité des liens qu'il établit progressivement avec le sujet. En effet, le chercheur qui situe sa posture dans le paradigme interprétatif doit être en mesure de s'occuper des incidents qui peuvent nuire à l'authenticité des rapports avec les sujets et, par le fait même, réduire les bienfaits de leur participation à la recherche.

\section{Critères de rigueur de la recherche-action selon les modèles adoptés}

Van der Maren (1996) affirme que toute recherche interprétative doit d'abord respecter les critères de base de la recherche scientifique, notamment les critères méthodologiques et relationnels déjà mentionnés. II ajoute toutefois que chaque modèle de recherche a ses particularités et doit donc retenir les critères de rigueur qui lui sont propres. 
Par exemple, on admet que les critères de validité d'une recherche descriptive ne sont pas les mêmes que ceux d'une analyse interprétative ou d'une théorie de l'action : elles n'ont pas les mêmes fonctions, elles n'ont pas les mêmes prétentions et elles ne doivent pas ni ne peuvent pas être évaluées selon des critères identiques. (p. 1)

La recherche-action s'insère généralement dans un paradigme pragmatico-interprétatif (Savoie-Zajc, 2001) : interprétatif, car l'objectif est de comprendre le sens que les acteurs donnent à leur réalité, et pragmatique, car le savoir qui ressort de la recherche a des implications pratiques, utiles et concrètes dans leur vie professionnelle (Debuyist, Digneffe et Pires, 2008; Morgan, 2007). Elle se distingue des autres types de recherche interprétative selon trois composantes fondamentales, soit l'intention, les rôles et la démarche méthodologique. L'intention de la recherche-action est de produire un changement dans un milieu professionnel donné et d'améliorer les pratiques (Dolbec et Clément, 2000; Savoie-Zajc, 2001). Par ailleurs, sur le plan des rôles, la recherche-action implique les acteurs à titre de cochercheurs. En effet, Gauthier (1984) indique qu'il s'agit d'une « modalité de recherche qui rend l'acteur chercheur et qui fait du chercheur un acteur. » (p. 522). Ce rapport symétrique entre chercheurs et praticiens à toutes les étapes de la recherche fait en sorte que la production du savoir est validée dans l'action et dans la pratique. Enfin, pour que l'amélioration de la pratique soit possible, la recherche-action emprunte une démarche méthodologique de résolution de problèmes selon un cycle de planification, d'action, d'observation et de réflexion (Dolbec et Clément, 2000). En effet, ces trois composantes font en sorte que les critères de rigueur méthodo logique et les critères relationnels de la recherche interprétative ne suffisent pas pour assurer la rigueur de la recherche-action. C'est la raison pour laquelle Savoie-Zajc (2001) suggère quatre nouveaux critères qui tiennent compte des particularités de la recherche-action, soit les critères du respect des valeurs et des principes démocratiques, de faisabilité, d’appropriation ainsi que de cohérence systémique.

\section{Respect des valeurs et des principes démocratiques}

Le critère du respect des valeurs et des principes démocratiques s'associe au groupe de critères relationnels qui mettent de l'avant l'éthique des procédures (Gohier, 2004). Ce critère renvoie effectivement à l'attitude du chercheur par rapport aux sujets (cochercheurs) et à sa responsabilité de garantir la qualité des échanges, par exemple, en mettant en place un climat de travail propice à l'interaction et à la collaboration, en protégeant les droits des participants à s'exprimer librement et en s'assurant d'inclure tous les participants dans la prise de décision à toutes les étapes de la recherche (Dolbec et Clément, 2000; Savoie-Zajc, 2001). Pour vérifier et témoigner du respect des valeurs et des principes démocratiques dans la recherche, Savoie-Zajc (2001) suggère la tenue d'un journal de bord par tous les membres qui participent à la recherche ainsi que des rencontres périodiques d'objectivation afin de discuter ouvertement de situations qui émergent et d'apporter, au besoin, des ajustements au processus de recherche. 


\section{Faisabilité}

Toujours dans l'ordre de l'éthique des procédures, le critère de faisabilité renvoie à la pertinence de la recherche-action pour le groupe, à la planification d'un projet qui tient compte des contraintes du milieu et à la viabilité du changement ou des solutions que les résultats proposent (Savoie-Zajc, 2001). Dans ce contexte de résolution de problèmes, les solutions mises de l'avant doivent orienter les actions et les décisions des participants tout en tenant compte de la réalité du milieu. À quoi bon mettre sur pied un projet dont la question est peu pertinente pour le milieu? Pour quelle raison s'acharner à discuter de solutions qui ne pourront effectivement pas être mises en œuvre? Le critère de faisabilité exige que le projet soit centré sur un problème réel de la profession et que les solutions répondent aux particularités du milieu (p. ex. : économiques, culturelles, politiques). Pour assurer la faisabilité du projet, Savoie-Zajc (2001) recommande d'établir des liens étroits avec le milieu (p. ex. : dirigeants, administration) et de maintenir une communication ouverte avec celui-ci pour pouvoir obtenir l'information nécessaire permettant de prendre des décisions éclairées au regard de la faisabilité du projet et de la mise en œuvre des solutions. En maintenant une communication continue avec les partenaires au regard du projet, la pertinence de la recherche et la viabilité des résultats dans le milieu sont soutenues.

\section{Appropriation}

Le critère d'appropriation rappelle l'importance d'assurer l'engagement des sujets à poursuivre la démarche initiée par la recherche (Dolbec et Clément, 2000; Savoie-Zajc, 2001). Il s'aligne avec les critères d'authenticité déjà abordés qui visent l'élargissement des connaissances des sujets, l'amélioration de leurs pratiques et l'acquisition d'outils les habilitant à passer à l'action. Ces éléments font partie intégrante de la recherche-action et représentent un enjeu incontournable qui consiste à amener les participants à s'approprier les résultats et à leur proposer des occasions de poursuivre la réflexion entamée dans le cadre de la recherche. La finalité de la recherche-action ne s'arrête pas à la production de savoirs, mais s'étend aussi à produire un changement durable et à transformer la pratique professionnelle des sujets. Le critère d'appropriation traite de la qualité des changements, c'est-à-dire de l'apprentissage et des prises de conscience des individus concernés par la recherche et de l'incidence de la participation sur la pratique professionnelle, et ce, à long terme. Bien que le journal de bord tenu par les différents membres du groupe de recherche-action et des rencontres périodiques d'objectivation puissent soutenir la pertinence et la faisabilité des solutions dans le milieu, seul un suivi longitudinal de la part du chercheur principal ou encore d'un vérificateur externe pourra permettre de déterminer jusqu'à quel point les cochercheurs se sont approprié les résultats de la recherche dans le but d'améliorer la pratique professionnelle de façon durable. 


\section{Cohérence systémique}

Le critère de cohérence systémique s'ajoute aux critères méthodologiques et réfère à la cohérence de la démarche de recherche et à la rigueur de la collecte et de l'analyse des données (Savoie-Zajc, 2001). Il se rapproche du critère de crédibilité et de fiabilité qui demande des descriptions riches, claires et détaillées du processus de recherche, l'accès aux données brutes et la documentation des influences diverses qui ont pu avoir une influence sur les résultats. Le critère de cohérence systémique est le point d'ancrage de la recherche-action puisqu'il assure l'alignement de la méthodologie sur les objectifs visés, la collecte et l'analyse de données ainsi que les résultats. Il souligne également l'importance de documenter les rôles et les responsabilités des cochercheurs et garantit un processus rigoureux de collecte et d'analyse de données. Pour s'assurer de la cohérence systémique de la recherche, la triangulation s'avère encore une fois la stratégie la plus utile, notamment la triangulation des observateurs, où des pairs vérifient l'alignement de la méthodologie.

La recherche-action est une démarche complexe qui implique non seulement l'interaction avec des sujets agissant sur un terrain généralement difficile à comprendre et dont les propos ne sont pas faciles à traduire, mais également l'engagement au changement et à l'action dans des milieux qui peuvent parfois y résister. Dans ce contexte, la conception et la mise en œuvre d'une démarche méthodologie impliquant les cochercheurs et garante de validité est également complexe. Les critères de rigueur méthodologique et relationnelle dont il a été question dans cet article permettent de constituer une grille de lecture de la validité de la recherche-action. Le tableau 1 résume les divers critères de rigueur tout en éliminant certaines redondances et suggère également des moyens à mettre en place pour soutenir des données et des résultats crédibles. Bien que le nombre de critères s'élève à onze, ils peuvent être regroupés selon trois composantes de la recherche-action : le savoir, le pouvoir et le vouloir (Pourtois, Desmet et Humbeeck, 2013) :

- la production d’un savoir qui est valide - les critères de crédibilité, de transférabilité, de fiabilité et de constance interne;

- le partage du pouvoir dans la recherche qui doit se faire dans le respect de l'autre et l'implication de tous - les critères d'équilibre et du respect des valeurs et des principes démocratiques;

- le vouloir agir pour instaurer l'amélioration durable de la pratique professionnelle - les critères d'authenticité ontologique, d'authenticité éducative, de faisabilité et d'appropriation qui englobent l'authenticité catalytique et tactique.

Au regard du nombre de critères de rigueur de la recherche-action et du nombre de mécanismes pour en témoigner, deux questions se posent:

1. À combien de critères de rigueur une recherche doit-elle obligatoirement satisfaire?

2. Combien de mécanismes doit-on intégrer à la recherche pour satisfaire à un critère de rigueur? 
Devant ces deux préoccupations, le point le plus important est la rigueur méthodologique et relationnelle à toutes les étapes de la recherche, de la méthode à la présentation des résultats. On peut difficilement penser, par exemple, que la faisabilité d'une recherche-action est plus importante que la crédibilité. En effet, il faut documenter suffisamment toutes les phases de la recherche pour satisfaire à la plupart, sinon à tous les critères de rigueur méthodologique et relationnelle. Bien qu'il existe des protocoles bien établis en recherche, il n'en demeure pas moins que le jugement du chercheur est primordial en recherche interprétative et que la charge de la preuve de rigueur lui revient ultimement. 


\section{Tableau 1 : Critères et moyens pour juger la rigueur de la recherche-action}

\begin{tabular}{|c|c|c|}
\hline Critères & Questionnement possible & Exemples de mécanismes pour satisfaire au critère \\
\hline Crédibilité & $\begin{array}{l}\text { - Les sujets se retrouvent-ils dans le texte de la } \\
\text { recherche? } \\
\text { - Est-ce que la méthodologie est décrite de façon } \\
\text { assez détaillée? } \\
\text { - Est-ce que l'analyse des données est le résultat } \\
\text { d'une démarche rigoureuse? }\end{array}$ & $\begin{array}{l}\text { - Retour aux sujets pour valider le texte de la recherche; } \\
\text { - Engagement prolongé dans le milieu pour s'approprier la culture du milieu et } \\
\text { comprendre le contexte; } \\
\text { - Description riche et détaillée de la méthodologie et des } \\
\text { méthodes. }\end{array}$ \\
\hline Transférabilité & $\begin{array}{l}\text { - Est-ce que le milieu est décrit de façon suffisante } \\
\text { pour que celui qui cherche à utiliser les résultats de } \\
\text { la recherche puisse faire des liens avec son propre } \\
\text { milieu? } \\
\text { - Est-ce que les sujets qui participent à la recherche } \\
\text { sont clairement décrits? }\end{array}$ & $\begin{array}{l}\text { - Description riche et détaillée de l'école et de son contexte; } \\
\text { - Description détaillée des sujets de la recherche. }\end{array}$ \\
\hline Fiabilité & $\begin{array}{l}\text { - Le chercheur est-il conscient de ses biais? } \\
\text { - La recherche est-elle exempte de préjugés? }\end{array}$ & $\begin{array}{l}\text { - Journal de bord du chercheur pour mettre en évidence la } \\
\text { réflexion sur les biais, les présupposés; } \\
\text { - Implication de plus d'un chercheur à toutes les étapes de la } \\
\text { recherche (p. ex. : vérification intercodeurs); } \\
\text { - Triangulation des sources de données (p. ex. : entretien semi- } \\
\text { dirigé, observation, analyse de documents) pour rendre compte d'un même } \\
\text { phénomène. }\end{array}$ \\
\hline Constance interne & $\begin{array}{l}\text { - Les données sont-elles accessibles (p. ex., enregis- } \\
\text { trements, transcription d'entretiens)? } \\
\text { - Les données ont-elles été recueillies avec des } \\
\text { procédures rigoureuses? }\end{array}$ & $\begin{array}{l}\text { - Description riche et détaillée des procédures de collecte de données; } \\
\text { - Journal de bord pour documenter l'évolution de la recherche et les justifications } \\
\text { des décisions (p. ex. : raffinement des questions de recherche); } \\
\text { - Triangulation des chercheurs à toutes les étapes de la recherche (p. ex. : collecte } \\
\text { de données). }\end{array}$ \\
\hline $\begin{array}{l}\text { Cohérence } \\
\text { systémique }\end{array}$ & $\begin{array}{l}\text { - Est-ce que la démarche de la recherche est } \\
\text { cohérente (p. ex. : alignement entre la méthodolo- } \\
\text { gie, les objectifs visés, la collecte et l'analyse des } \\
\text { données)? } \\
\text { - Est-ce que les rôles et responsabilités des membres } \\
\text { sont documentés? }\end{array}$ & $\begin{array}{l}\text { - Vérification externe dans le but de porter un jugement sur la } \\
\text { démarche de la recherche; } \\
\text { - Justification des instruments de collecte de données; } \\
\text { - Documentation des rencontres (p. ex. : agenda, procès-verbaux ou comptes } \\
\text { rendus); } \\
\text { - Description riche et détaillée des procédures de collecte et d'analyse de don- } \\
\text { nées. }\end{array}$ \\
\hline
\end{tabular}




\begin{tabular}{|c|c|c|}
\hline Critères & Questionnement possible & Exemples de mécanismes pour satisfaire au critère \\
\hline Équilibre & $\begin{array}{l}\text { - Est-ce que tous les sujets ont eu l'occasion d'expri- } \\
\text { mer leurs idées pendant la recherche? } \\
\text { - Est-ce que les points de vue de l'ensemble des } \\
\text { sujets sont exprimés de façon équitable dans la } \\
\text { recherche? }\end{array}$ & $\begin{array}{l}\text { - Retour aux sujets pour valider le texte de la recherche; } \\
\text { - Engagement prolongé dans le milieu pour s'approprier la culture du milieu et } \\
\text { comprendre le contexte; } \\
\text { - Implication de plus d'un chercheur à toutes les étapes de la } \\
\text { recherche. }\end{array}$ \\
\hline $\begin{array}{l}\text { Authenticité } \\
\text { ontologique }\end{array}$ & $\begin{array}{l}\text { - Les sujets ont-ils amélioré leurs connaissances et } \\
\text { élargi leurs représentations à propos de l'objet ou } \\
\text { du phénomène à l'étude? } \\
\text { - Les sujets ont-ils des représentations plus riches } \\
\text { et plus complexes de l'objet ou du phénomène à } \\
\text { l'étude? }\end{array}$ & $\begin{array}{l}\text { - Témoignages des participants attestant de l'élargissement } \\
\text { de leurs connaissances et de l'amélioration de leur pratique } \\
\text { professionnelle. }\end{array}$ \\
\hline $\begin{array}{l}\text { Authenticité } \\
\text { éducative }\end{array}$ & $\begin{array}{l}\text { - Les sujets ont-ils approfondi leur connaissance } \\
\text { d'eux-mêmes? } \\
\text { - Les sujets ont-ils une meilleure compréhension des } \\
\text { représentations que se font les autres enseignants? }\end{array}$ & - Témoignages des participants attestant de l'élargissement de leurs perspectives. \\
\hline $\begin{array}{l}\text { Respect des valeurs } \\
\text { et des principes } \\
\text { démocratiques }\end{array}$ & $\begin{array}{l}\text { - Est-ce que les droits des membres du groupe sont } \\
\text { respectés? } \\
\text { - Le climat des rencontres est-il propice aux } \\
\text { échanges? } \\
\text { - Est-ce que tous les membres du groupe sont impli- } \\
\text { qués dans la prise de décisions de la recherche? }\end{array}$ & $\begin{array}{l}\text { - Journal de bord des cochercheurs; } \\
\text { - Rencontres d’objectivation; } \\
\text { - Vérification externe. }\end{array}$ \\
\hline Faisabilité & $\begin{array}{l}\text { Est-ce que la recherche et les solutions tiennent } \\
\text { compte des contraintes du milieu scolaire (p. ex., } \\
\text { contraintes sociales, politiques, économiques, } \\
\text { culturelles)? }\end{array}$ & $\begin{array}{l}\text { - Établissement de lien avec le milieu (p. ex. : partenaires, } \\
\text { dirigeants) pour vérifier la pertinence du projet et la viabilité des solutions dans } \\
\text { le milieu. }\end{array}$ \\
\hline $\begin{array}{l}\text { Appropriation } \\
\text { Authenticité } \\
\text { catalytique } \\
\text { Authenticité tactique }\end{array}$ & $\begin{array}{l}\text { - Est-ce que tous les membres se sont pleinement } \\
\text { engagés dans la recherche? } \\
\text { - Les membres ont-ils les outils nécessaires pour } \\
\text { passer à l'action? } \\
\text { - Les sujets ont-ils poursuivi la réflexion après la } \\
\text { recherche? }\end{array}$ & $\begin{array}{l}\text { - Diffusion et présentation du rapport de recherche par les cochercheurs; } \\
\text { - Suivi longitudinal de la part du chercheur principal auprès des membres du } \\
\text { groupe; } \\
\text { - Vérification externe. }\end{array}$ \\
\hline
\end{tabular}




\section{Références}

Anadón, M. (2006). La recherche « qualitative ». De la dynamique de son évolution aux acquis indéniables et aux questionnements présents. Recherches qualitatives, 26(1), 5-31.

Cohen, L., Manion L. et Morrison, K. (2011). Validity and reliability. Dans L. Cohen, L. Manion et K. Morrison (dir.), Research Methods in Education (p. 179-216). London, New York: Routledge.

Creswell, J. W. (2007). Qualitative inquiry and research design: Choosing among five traditions. Thousand Oaks, CA: Sage Publications.

Denzin, N. K. et Lincoln, Y. S. (2005). Introduction: The discipline and practice of qualitative research. Dans N. K. Denzin et Y. S. Lincoln (dir.), The SAGE handbook of qualitative research (3e éd., p. 1-32). Thousand Oaks, CA : Sage.

Dolbec, A. et Clément, J. (2000). La recherche-action. Dans L. Savoie-Zajc (dir.), Introduction à la recherche en éducation (p. 199-224). Sherbrooke : Éditions du CRP.

Gauthier, B. (1984). Recherche sociale. Québec: Presses de l’Université du Québec.

Gohier, C. (2004). De la démarcation entre critères d'ordre scientifique et d'ordre éthique en recherche interprétative. Recherches qualitatives, 24, 3-17.

Guba, E. G. (1981). Criteria for assessing the trustworthiness of naturalistic inquiries. Educational Communication and Technology Journal, 29(2), 75-91.

Guba, E. G. et Lincoln, Y. S. (1989). Judging the quality of fourth generation evaluation. Dans E. G. Guba et Y. S. Lincoln (dir.), Fourth Generation Evaluation (p. 229-251). London : Sage.

Guba, E. G. et Lincoln, Y. S. (2005). Paradigmatic controversies and emerging confluences. Dans N. K. Denzin, et Y. S. Lincoln (dir.), The SAGE handbook of qualitative research (3 éd., p. 191-216). Thousand Oaks, CA : Sage.

Kolbe, R. H. et Burnett, M. S. (1991). Content-analysis research: An examination of applications with directives for improving research reliability and objectivity. Journal of Consumer Research, 18, 243-250.

Laperrière, A. (1997). Les critères de scientificité des méthodes qualitatives. Dans J. Poupart, J.-P. Deslauriers, L.-H. Groulx, A. Laperrière, R. Mayer et A. Pires (dir.), La recherche qualitative. Enjeux épistémologiques et méthodologiques (p. 365-388). Montréal : Gaëtan Morin.

Lincoln, Y. S. (1995). Emerging criteria for quality in qualitative and interpretive research. Qualitative Inquiry, 1(3), 275-289.

Lincoln, Y. S. et Guba, E. G. (1985). Naturalistic inquiry. Beverly Hills CA: Sage.

Manning, K. (1997). Authenticity in constructivist inquiry : Methodological considerations without prescriptions. Qualitative Inquiry, 3(1), 93-115. 
Miles, M. B. et Huberman, M. A. (2003). Analyse des données qualitatives (2éd.). Paris : De Boeck.

Mucchielli, A. (2009). Dictionnaire des méthodes qualitatives en sciences humaines ( $3^{e}$ éd.). Paris: Armand Colin.

Pourtois, J.-P. et Desmet, H. (2007). Épistémologie et instrumentation en sciences humaines (3e éd.). Wavre, Belgique : Mardaga.

Pourtois, J.-P., Desmet, H. et Humbeeck, B. (2013). La recherche-action, un instrument de compréhension et de changement du monde. Recherches qualitatives - Hors Série, 5, 99-111.

Savoie-Zajc, L. (2000). La recherche qualitative/interprétative en éducation. Dans T. Karsenti et L. Savoie-Zajc (dir.), Introduction à la recherche en éducation (p. 171-198). Sherbrooke : Éditions du CRP.

Savoie-Zajc, L. (2001). La recherche-action en éducation : ses cadres épistémologiques, sa pertinence, ses limites. Dans M. Anadón et M. L'Hostie (dir.), Nouvelles dynamiques de recherche en éducation (p. 15-49). Sainte-Foy: Les Presses de l'Université Laval.

Savoie-Zajc, L. (2011). La recherche qualitative/interprétative en éducation. Dans T. Karsenti et L. Savoie-Zajc (dir.), La recherche en éducation (p. 123-146). Saint-Laurent : Éditions RPI.

Van der Maren, J.-M. (1996, octobre). La recherche qualitative peut-elle être rigoureuse? Communication présentée au Colloque international Recherche qualitative et gestion, HEC, Montréal, Canada. 